\title{
SETS OF RECURRENCE AND GENERALIZED POLYNOMIALS
}

\author{
VitAly BERGELSON \\ AND \\ INGER JOHANNE HÅLAND
}

\section{INTRODUCTION}

A set $S \subset \mathbb{Z}$ is called a set of recurrence if for any invertible measure preserving system $(X, \mathcal{B}, \mu, T)$ and any $A \in \mathcal{B}$ with $\mu(A)>0$ there exists $n \in S, n \neq 0$, such that $\mu\left(A \cap T^{-n} A\right)>0$. For example, for any infinite $E \subset \mathbb{N}$, the set of differences $E-E=\{x-y \mid x, y \in E, x>y\}$ is a set of recurrence. Another simple example of a set of recurrence is given by any set containing arbitrarily long arithmetic progressions of the form $\{a, 2 a, \cdots, k a\}$. Less trivial example is the following. Let $p(t)$ be a polynomial taking integer values on integers and satisfying $p(0)=0$. Then $\{p(n) \mid n \in \mathbb{Z}\}$ is a set of recurrence. This result is due, independently, to $\mathrm{H}$. Furstenberg [7] and A. Sárközy [13]. One can show that if a polynomial $p(t)$ takes integer values on integers then the set $\{p(n) \mid n \in \mathbb{Z}\}$ is a set of recurrence if and only if it contains a multiple of $a$ for any $a \in \mathbb{N}$. (See, for example, [12].)

The notion of a set of recurrence was introduced by H. Furstenberg in connection with number theoretical applications. (See, for example [7] and [6].) The following brief remarks give some necessary background related to this connection.

For a subset $E \subset \mathbb{Z}$ its upper Banach density is defined by

$$
d^{*}(E)=\limsup _{|I| \rightarrow \infty} \frac{|E \cap I|}{|I|},
$$

where $I$ ranges over all intervals of $\mathbb{Z}$.

The upper density of $E, \bar{d}(E)$ is defined by

$$
\bar{d}(E)=\limsup _{n \rightarrow \infty} \frac{|E \cap[-n, n]|}{2 n+1} .
$$

(If $E \subset \mathbb{N}$ one puts $\bar{d}(E)=\lim \sup _{n \rightarrow \infty} \frac{|E \cap[1, n]|}{n}$.)

If the limit in question exists, we say that $E$ has density and denote it by $d(E)$.

Furstenberg's correspondence principle (cf. [5,7], see also [2]). Let $E \subset \mathbb{Z}$ with $d^{*}(E)>0$. Then there exist a measure preserving system $(X, \mathcal{B}, \mu, T)$ and a set $A_{E} \in \mathcal{B}$ with $\mu\left(A_{E}\right)=d^{*}(E)$ such that for any $k \in \mathbb{N}$ and for any $n_{1}, n_{2}, \ldots, n_{k} \in \mathbb{Z}$ one has:

$$
\mu\left(A_{E} \cap T^{-n_{1}} A_{E} \cap \cdots \cap T^{-n_{k}} A_{E}\right) \leq d^{*}\left(E \cap\left(E-n_{1}\right) \cap \cdots \cap\left(E-n_{k}\right)\right) .
$$

The following corollary of Furstenberg's correspondence principle makes the connection between sets of recurrence and number theory quite apparent (cf. [3], see also [2, Theorem 4.4] for a stronger result.) 
Proposition 1.1. A set $R \subset \mathbb{Z}$ is a set of recurrence if and only if for any $E \subset \mathbb{Z}$ with $d^{*}(E)>0$ one has

$$
(E-E) \cap R \neq \emptyset .
$$

In other words, $R$ is a set of recurrence if and only if one can always solve the diophantine equation $x-y=z$ with $x, y \in E, z \in R$. Before giving the proof of Proposition 1.1 we formulate a convenient lemma (which will also be utilized in the proof of Proposition 1.3 (the uniformity of recurrence) below.

Lemma 1.2 [1]. Let $(X, \mathcal{B}, \mu, T)$ be a measure preserving system and let $A \in \mathcal{B}$, $\mu(A)=a>0$. Then there exists an increasing sequence $\left\{n_{m}\right\}_{m=1}^{\infty}$ with $d\left(\left\{n_{m}\right\}\right) \geq$ $a$ such that for any $m \in \mathbb{N}$ one has

$$
\mu\left(A \cap T^{-n_{1}} A \cap \cdots \cap T^{-n_{m}} A\right)>0 .
$$

Proof of Proposition 1.1. In one direction the result follows immediately from Furstenberg's correspondence principle. Indeed, if $E \subset \mathbb{Z}, d^{*}(E)>0$, then for any $n$, and in particular for any $n \in R$, one has $d^{*}(E \cap(E-n)) \geq \mu\left(A_{E} \cap T^{-n} A_{E}\right)$. If $R$ is a set of recurrence one can find $n \in R$ such that $\mu\left(A_{E} \cap T^{-n} A_{E}\right)>0$. Then $d^{*}(E \cap(E-n))>0$ and, in particular, $E \cap(E-n) \neq \emptyset$ which is equivalent to $(E-E) \cap R \neq \emptyset$.

Assume now that for any $E \subset \mathbb{Z}$ with $d^{*}(E)>0$ one has $(E-E) \cap R \neq \emptyset$. We shall show that $R$ is a set of recurrence. Let $(X, \mathcal{B}, \mu, T)$ be a measure preserving system and $A \in \mathcal{B}$ with $\mu(A)>0$. By Lemma 1.2 there exists a set $E=\left\{n_{m}\right\}_{m=1}^{\infty}$ with $d(E)>0$ such that for any $n_{1}, n_{2} \in E, \mu\left(T^{-n_{1}} A \cap T^{-n_{2}} A\right)>0$ which implies $\mu\left(A \cap T^{-\left(n_{2}-n_{1}\right)} A\right)>0$. Since $(E-E) \cap R \neq \emptyset$, there exist distinct $n_{1}, n_{2} \in E$, $n \in R$ with $n_{1}-n_{2}=n$. This gives $\mu\left(A \cap T^{-n} A\right)>0$. We are done.

Proposition 1.3 (Uniformity of recurrence). If $R \subset \mathbb{Z}$ is a set of recurrence, then for any $a>0$ there exists $K=K(a)$, such that for any measure preserving system $(X, \mathcal{B}, \mu, T)$ and $A \in \mathcal{B}$ with $\mu(A)>a$ there exists $n \in R$ with $|n|<K$ satisfying $\mu\left(A \cap T^{-n} A\right)>0$.

Proof. Let us show first that for any $a>0$ there exists $L=L(a)$ such that if $I=[N+1, \ldots, M]$ is any interval in $\mathbb{Z}$ with $|I|=M-N>L$ and $F \subset I$ is any subset in $I$ satisfying $\frac{|F \cap I|}{|I|}>a$, then there exist $x, y \in F$ and $k \in R$ with $x-y=k$ (which is the same as $(F-F) \cap R \neq \emptyset$ ). Indeed, if this was not the case, one could find arbitrarily long intervals $I_{n}$ and subsets $F_{n} \subset I_{n}$ satisfying $\frac{\left|F_{n} \cap I_{n}\right|}{\left|I_{n}\right|}>a$ but such that $\left(F_{n}-F_{n}\right) \cap R=\emptyset$. Let $\left|I_{n}\right|=N_{n}$ and let

$\mathcal{F}_{n}(b)=\left\{F \subset I \mid I=\left\{m+1, \ldots, m+N_{n}\right\}, m \in \mathbb{Z}, \frac{|F \cap I|}{|I|}>b,(F-F) \cap R=\emptyset\right\}$.

Note that $\mathcal{F}_{n}(b)$ is a shift-invariant family, i.e. if $F \in \mathcal{F}_{n}(b)$ then also $F-m=$ $\{a-m \mid a \in F\} \in \mathcal{F}_{n}(b)$ for any $m \in \mathbb{Z}$. Note also that $\mathcal{F}_{n}(a) \subset \mathcal{F}_{n}(b)$ for all $0<b<a$ and that $\mathcal{F}_{n}(a) \neq \emptyset$ for all $n$. Let $0<a_{0}<a$, and let $N_{n+1}=l_{n} N_{n}+r_{n}$, where $0 \leq r_{n}<N_{n}$. By going to a subsequence of $N_{n}, n=1,2, \ldots$, if necessary, one may assume that $l_{n}$ are sufficiently large so that $\sum_{n=1}^{\infty} 1 / l_{n}<a-a_{0}$. Let 
$\varepsilon_{n}=\sum_{i=1}^{n} 1 / l_{i}$, and let $F \in \mathcal{F}_{n+1}\left(a_{0}+\varepsilon_{n}\right), F \subset I_{n+1}$. There exists a subinterval $J \subset I_{n+1},|J|=N_{n}$, such that $\frac{|F \cap J|}{|J|}>a_{0}+\varepsilon_{n}-1 / l_{n}=a_{0}+\varepsilon_{n-1}$. Hence, $F \cap J \in \mathcal{F}_{n}\left(a_{0}+\varepsilon_{n-1}\right) \subset \mathcal{F}_{n}\left(a_{0}\right)$. So for any $k \in \mathbb{N}$, there exist sets $G_{n} \in \mathcal{F}_{n}\left(a_{0}+\right.$ $\left.\varepsilon_{n-1}\right) \subset \mathcal{F}_{n}\left(a_{0}\right), n=1, \ldots, k$, such that $G_{1} \subset \cdots \subset G_{n} \subset G_{n+1} \subset \cdots \subset G_{k}$. If $G_{1}=\left\{m+1, \ldots, m+N_{1}\right\}$, then $G_{1}-m \subset\left\{1, \ldots, N_{1}\right\}, G_{n}-m \in \mathcal{F}_{n}\left(a_{0}\right), n=$ $1, \ldots, k$, and $\left(G_{1}-m\right) \subset \cdots \subset\left(G_{n}-m\right) \subset\left(G_{n+1}-m\right) \subset \cdots \subset\left(G_{k}-m\right)$. Since there are only finitely many sets $F \subset\left\{1, \ldots, N_{1}\right\}$, there exists an infinite sequence $G_{1} \subset \cdots \subset G_{n} \subset G_{n+1} \subset \cdots$, where $G_{n} \in \mathcal{F}_{n}\left(a_{0}\right)$ for each $n$. By taking $E=\bigcup_{n=1}^{\infty} G_{n}$, one gets a set $E$ with $\bar{d}(E) \geq a_{0}$ such that $(E-E) \cap R=\emptyset$ which contradicts Proposition 1.1.

Now let $(X, \mathcal{B}, \mu, T)$ be a measure preserving system and let $A \in \mathcal{B}$ with $\mu(A)>$ $a>0$. By Lemma 1.2 there exists a set $S=\left\{n_{i}\right\}_{i=1}^{\infty}$ with $d(S) \geq \mu(A)>a$ and such that $\mu\left(A \cap T^{-n_{1}} A \cap T^{-n_{2}} A \cap \cdots \cap T^{-n_{k}} A\right)>0$ for any $k \in \mathbb{N}$. Let $I=[N+1, \ldots, M]$ be an interval in $\mathbb{Z}$ with $2 L>|I|>L$ and such that $\frac{|S \cap I|}{|I|}>a$ (since $d(S)>a$, this is always possible). Then for some $n_{1}, n_{2} \in S \cap I, n \in R$ one has $n_{1}-n_{2}=n$. This gives $\mu\left(T^{-n_{1}} A \cap T^{-n_{2}} A\right)=\mu\left(A \cap T^{-n} A\right)>0$ with $|n|<2 L$. This shows that one can put $K(a)=2 L(a)$. We are done.

Remarks. (i) One can find another proof of Proposition 1.3 in [4].

(ii) So far we have only treated subsets of $\mathbb{Z}$ and $\mathbb{Z}$-actions. However, everything extends to the $\mathbb{Z}^{k}$-case. Let us say that a set $E \subset \mathbb{Z}^{k}$ has positive upper density $\bar{d}(E)$ if

$$
\bar{d}(E)=\limsup _{N \rightarrow \infty} \frac{\left|E \cap\{-N, \ldots, N\}^{k}\right|}{(2 N+1)^{k}}>0 .
$$

A set $R \subset \mathbb{Z}^{k}$ is a set of recurrence if for any probability space $(X, \mathcal{B}, \mu)$, any commuting invertible measure preserving transformations $T_{1}, T_{2}, \ldots, T_{k}$ and any $A \in \mathcal{B}$ with $\mu(A)>0$, there exists $r=\left(r_{1}, \ldots, r_{k}\right) \in R, r \neq(0, \ldots, 0)$, such that $\mu\left(A \cap T_{r}^{-1} A\right)>0$, where $T_{r}=T_{1}^{r_{1}} T_{2}^{r_{2}} \cdots T_{k}^{r_{k}}$.

Note that the $\mathbb{Z}^{k}$-version of Proposition 1.3 (uniformity of recurrence) is proved completely analogously to the case $k=1$.

(iii) See Proposition 2.1 below.

Corollary 1.4. For any fixed $k \in \mathbb{N}$, any sequence $\left\{b_{i}\right\}_{i=1}^{\infty} \subset \mathbb{Z}$ and any increasing sequence of integers $\left\{N_{i}\right\}_{i=1}^{\infty}$ the set $R=\bigcup_{i=1}^{\infty}\left\{b_{i}, 2^{k} b_{i}, 3^{k} b_{i}, \ldots, N_{i}^{k} b_{i}\right\}$ is a set of recurrence.

Proof. Given an arbitrary measure preserving system $(X, \mathcal{B}, \mu, T)$ and $A \in \mathcal{B}$ with $\mu(A)>0$ we have to show that there exists $n \in R$ with $\mu\left(A \cap T^{-n} A\right)>0$. For the set of recurrence $\left\{n^{k}\right\}_{n=1}^{\infty}$ and for any $a$ satisfying $\mu(A)>a>0$ let $K=K(a)$ as defined in the formulation of Proposition 1.3. Let $i$ be such that $N_{i}>K$. Applying the uniformity of recurrence to the system $(X, \mathcal{B}, \mu, S)$ where $S=T^{b_{i}}$ and to $\left\{n^{k}\right\}_{n=1}^{\infty}$, we get $n, 1 \leq n \leq N_{i}$ such that $\mu\left(A \cap T^{-b_{i} n^{k}} A\right)=\mu\left(A \cap S^{-n^{k}} A\right)>0$.

From this corollary it follows that, for example, $\left\{\left[\alpha n^{2}\right] \mid n \in \mathbb{N}\right\}$ is a set of recurrence. To see this, notice that for any $x \in \mathbb{R}$ and $h \in \mathbb{N}$, we have the following 
identity

$$
[h x]=h[x]+\sum_{i=0}^{h-1} i 1_{\left[\frac{i}{h}, \frac{i+1}{h}\right)}(\{x\}),
$$

so that $[h x]=h[x]$ if and only if $h<\frac{1}{\{x\}}$. Let $\left\{m_{i}\right\}_{i=1}^{\infty} \subset \mathbb{N}$ be a sequence such that $\left\{\alpha m_{i}^{2}\right\} \rightarrow 0$, and let $b_{i}=\left[\alpha m_{i}^{2}\right]$ and $N_{i}=\left[1 /\left\{\alpha m_{i}^{2}\right\}^{1 / 2}\right]-1$. Then

$$
\left[\alpha\left(n m_{i}\right)^{2}\right]=\left[\alpha m_{i}^{2}\right] n^{2}=b_{i} n^{2} \quad \text { for } \quad n=1,2, \ldots, N_{i} .
$$

Hence, $\left\{\left[\alpha n^{2}\right] \mid n \in \mathbb{N}\right\}$ is a set of recurrence by Corollary 1.4. To treat more complicated expressions like $\left[\alpha n^{2}\right]+[\beta n]$ one needs a little more elaborated technique, which will be developed in Section 2.

In this paper we shall exploit the so called generalized polynomials to give some new examples of and answer some questions about sets of recurrence.

Generalized polynomials form a natural family of functions which are obtained from polynomials by the use of the greatest integer function [·], addition and multiplication. For example, $[\alpha x] \beta x^{2}$ or $[\alpha x][\beta x]+\left[[\delta x]\left[\epsilon x^{3}\right]^{2} \eta\right]$ are generalized polynomials. A few words of caution are in place here. First of all, a generalized polynomial can have different symbolic representations. For example, $[(1+\alpha) n] \beta n=\beta n^{2}+[\alpha n] \beta n$. This will not cause any confusion since we are going to be interested in the values taken by generalized polynomials. Second, on many occasions we shall not distinguish between the set of values of a generalized polynomial, $\{q(n) \mid n \in \mathbb{N}\}$, and the naturally formed sequence $(q(n))_{n=1}^{\infty}$. The "sequential" vision of generalized polynomials will be especially natural when we shall be checking whether the set $\{q(n) \mid n \in \mathbb{N}\}$ is an averaging set of recurrence.

Definition 1.5. An ordered set of recurrence $\left\{x_{n} \mid n \in \mathbb{N}\right\} \subset \mathbb{Z}$ is an averaging set of recurrence if for any invertible measure preserving system $(X, \mathcal{B}, \mu, T)$ and any $A \in \mathcal{B}$ with $\mu(A)>0$ one has

$$
\lim _{N \rightarrow \infty} \frac{1}{N} \sum_{n=1}^{N} \mu\left(A \cap T^{-x_{n}} A\right)>0 .
$$

For example, any set of density one in $\mathbb{N}$ is an averaging set of recurrence. On the other hand, one can easily construct an infinite set $E \subset \mathbb{N}$ such that the naturally ordered set of recurrence $\{x-y \mid x, y \in E, x>y\}$ is not an averaging set of recurrence.

Given a polynomial $p(n)$ which takes integer values on integers one can show that $p(\mathbb{N})$ is a set of recurrence if and only if it is an averaging set of recurrence (and if and only if $p(\mathbb{N}) \cap a \mathbb{Z} \neq \emptyset$ for all $a \in \mathbb{N}$ ). The criteria for when a set $q(\mathbb{N})$ is a set of recurrence, where $q(n)$ is a generalized polynomial, are more complicated. Here are a few illustrative examples. Notice that a necessary condition for a set $R$ to be a set of recurrence is that $R \cap a \mathbb{Z} \neq \emptyset$ for any $a \in \mathbb{N}$. If $\alpha>1$ is irrational, then $\left[[2 \alpha n] \frac{1}{\alpha}\right]=\left[2 n-\{2 \alpha n\} \frac{1}{\alpha}\right]=2 n-1$, so that $\left\{\left[[2 \alpha n] \frac{1}{\alpha}\right] \mid n \in \mathbb{N}\right\}$ is not 
a set of recurrence. However, $\{[[\sqrt{2} n] \sqrt{2}] \mid n \in \mathbb{N}\} \cap a \mathbb{Z} \neq \emptyset$ for any $a \in \mathbb{N}$ but is not a set of recurrence. On the other hand, $\left\{[[\sqrt{2} n] \sqrt{2}]^{2} \mid n \in \mathbb{N}\right\}$ is an averaging set of recurrence. Curiously enough, both the sets $\{[[\sqrt{3} n] \sqrt{3}] \mid n \in \mathbb{N}\}$ and $\left\{[[\sqrt{3} n] \sqrt{3}]^{2} \mid n \in \mathbb{N}\right\}$ are not sets of recurrence. (See Prop. 4.1, (1) and (2)).

Conjecture. Let $q(n)$ be a generalized polynomial, taking integer values on integers. Then $q(\mathbb{N})$ is a set of recurrence if and only if it is an averaging set of recurrence.

Note that in order to prove that $x_{n}, n=1,2, \ldots$, is an averaging set of recurrence, it is enough to show two things:

(i) $\lim \sup _{N \rightarrow \infty} \frac{1}{N} \sum_{n=1}^{N} \mu\left(A \cap T^{-x_{n}} A\right)>0$ for any $(X, \mathcal{B}, \mu, T)$ and $A \in \mathcal{B}$ with $\mu(A)>0$,

(ii) $\lim _{N \rightarrow \infty} \frac{1}{N} \sum_{n=1}^{N} e^{2 \pi i x_{n} \lambda}$ exists for any $\lambda \in \mathbb{R}$.

(To see that (ii) implies the existence of $\lim _{N \rightarrow \infty} \frac{1}{N} \sum_{n=1}^{N} \mu\left(A \cap T^{-x_{n}} A\right)$ use the spectral theorem and Lebesgue convergence theorem:

$$
\begin{aligned}
\lim _{N \rightarrow \infty} \frac{1}{N} \sum_{n=1}^{N} \mu\left(A \cap T^{-x_{n}} A\right) & =\lim _{N \rightarrow \infty} \frac{1}{N} \sum_{n=1}^{N}\left\langle 1_{A}, T^{x_{n}} 1_{A}\right\rangle \\
& =\lim _{N \rightarrow \infty} \frac{1}{N} \sum_{n=1}^{N} \int_{0}^{1} e^{2 \pi i x_{n} \lambda} d \nu_{A}(\lambda)
\end{aligned}
$$

In light of this it would be nice to know the answer to the following:

Question. Is it true that the limit $\lim _{N \rightarrow \infty} \frac{1}{N} \sum_{n=1}^{N} e^{2 \pi i q(n) \lambda}$ exists for any generalized polynomial $q(n)$ and any $\lambda \in \mathbb{R}$ ?

In Sections 2 and 3 two different methods of studying sets of recurrence of the form $q(\mathbb{N})$ where $q$ is a generalized polynomial are presented. In Section 4 we give conditions for some concrete families of generalized polynomials to generate averaging sets of recurrence. A sample of results to be found in Section 4 was brought before Conjecture above.

\section{Generalized polynomials whose VALUES CONTAIN POLYNOMIAL PROGRESSIONS}

By a polynomial progression we mean a finite sequence of the form $p(n), n=$ $1,2, \ldots, N$, where $p(t)$ is a polynomial. In what follows we shall need a generalization of Corollary 1.4. We shall obtain this generalization as a corollary of the following. 
Proposition 2.1. Let $p_{1}(n), p_{2}(n), \ldots, p_{k}(n)$ be any polynomials taking integer values on integers and satisfying $p_{i}(0)=0, i=1,2, \ldots, k$. For any $a>0$ there exist positive constants $C(a)$ and $c(a)$ such that for any probability space $(X, \mathcal{B}, \mu)$, any commuting invertible measure preserving transformations $T_{1}, T_{2}, \ldots, T_{k}$ of $(X, \mathcal{B}, \mu)$ and any set $A \in \mathcal{B}$ with $\mu(A)>a$ there exists $n \in \mathbb{N}, n<C(a)$, such that

$$
\mu\left(A \cap T_{n} A\right)>c(a)
$$

where $T_{n}=T_{1}^{p_{1}(n)} T_{2}^{p_{2}(n)} \cdots T_{k}^{p_{k}(n)}$.

Proof. Let $(X, \mathcal{B}, \mu)$ be a probability space, $T_{1}, T_{2}, \ldots, T_{k}$ measure preserving transformations and let $A \in \mathcal{B}$ with $\mu(A)>a>0$. By a $\mathbb{Z}^{k}$-version of Furstenberg's correspondence principle it follows ([B1, Cor. 4.2.1]) that for any $E \subset \mathbb{Z}^{k}$ with $\bar{d}(E)>0$ one has

$$
(E-E) \cap P \neq \emptyset
$$

where $P=\left\{\left(p_{1}(n), p_{2}(n), \ldots, p_{k}(n)\right) \mid n \in \mathbb{Z}\right\}$. By Proposition 1.3 and the Remark after it, one sees that the following form of this statement is true: For any $a>0$ there exists $L=L(a)$ such that if $I=\prod_{i=1}^{k}\left[a_{i}, b_{i}\right] \subset \mathbb{Z}^{k}$ with $\min _{i}\left(b_{i}-a_{i}\right) \geq L$, then for any $F \subset I$ with $\frac{|F \cap I|}{|I|} \geq a / 2$ one has $(F-F) \cap P \neq \emptyset$. Consider now

$$
f(x)=\frac{1}{L^{k}} \sum_{1 \leq n_{1}, n_{2}, \ldots, n_{k} \leq L} 1_{A}\left(T_{1}^{n_{1}} T_{2}^{n_{2}} \cdots T_{k}^{n_{k}} x\right) .
$$

Let

$$
B=\{x \mid f(x) \geq a / 2\} .
$$

Since $0 \leq f(x) \leq 1$ and $\int f(x) d \mu=\mu(A) \geq a$, it follows that $\mu(B) \geq a / 2$. But for $x \in B, f(x) \geq a / 2$ implies that the set

$$
F=\left\{\left(n_{1}, n_{2}, \ldots, n_{k}\right) \in \mathbb{Z}^{k} \mid 1 \leq n_{i} \leq L, i=1,2, \ldots, k, x \in T_{1}^{-n_{1}} T_{2}^{-n_{2}} \cdots T_{k}^{-n_{k}} A\right\}
$$

satisfies $\frac{|F \cap I|}{|I|} \geq a / 2$, where $I=[1, L]^{k}$, and hence $(F-F) \cap P \neq \emptyset$. So

$$
B \subset \bigcup\left(T_{1}^{-n_{1}} T_{2}^{-n_{2}} \cdots T_{k}^{-n_{k}} A \cap T_{1}^{-\left(n_{1}+p_{1}(n)\right)} T_{2}^{-\left(n_{2}+p_{2}(n)\right)} \cdots T_{k}^{-\left(n_{k}+p_{k}(n)\right)} A\right)
$$

where the union is taken over all $\left(n_{1}, n_{2}, \ldots, n_{k}\right) \in \mathbb{Z}^{k}$ and $n \in \mathbb{Z}$ such that both vectors $\left(n_{1}, n_{2}, \ldots, n_{k}\right)$ and $\left(n_{1}+p_{1}(n), n_{2}+p_{2}(n), \ldots, n_{k}+p_{k}(n)\right)$ belong to $I$. But the number of such pairs does not exceed $L^{k+1}$. So one of the intersections

$$
\left(T_{1}^{-n_{1}} T_{2}^{-n_{2}} \cdots T_{k}^{-n_{k}} A \cap T_{1}^{-\left(n_{1}+p_{1}(n)\right)} T_{2}^{-\left(n_{2}+p_{2}(n)\right)} \cdots T_{k}^{-\left(n_{k}+p_{k}(n)\right)} A\right)
$$

has measure at least $\frac{a}{2 L^{k+1}}$.

Analogously to Corollary 1.4 we get 
Corollary 2.2. Let $p_{1}(n), p_{2}(n), \ldots, p_{k}(n)$ be any linearly independent polynomials taking integer values on integers, and satisfying $p_{i}(0)=0, i=1,2, \ldots, k$. For any integer-valued sequences $\left\{b_{1 i}\right\}_{i=1}^{\infty}, \ldots,\left\{b_{k i}\right\}_{i=1}^{\infty}$ and any increasing sequence of integers $\left\{N_{i}\right\}_{i=1}^{\infty}$, the set

$$
R=\bigcup_{i=1}^{\infty}\left\{\sum_{j=1}^{k} b_{j i} p_{j}(n) \mid n=1,2, \ldots, N_{i}\right\}
$$

is a set of recurrence.

As in the example after Corollary 1.4 it now follows that $\left\{\left[\alpha n^{2}\right]+[\beta n] \mid n \in \mathbb{Z}\right\}$ is a set of recurrence. By the following theorem it is also an averaging set of recurrence.

We call $R=\left\{x_{n} \mid n \in \mathbb{N}\right\}$ an almost averaging set of recurrence if for any measure preserving system $(X, \mathcal{B}, \mu, T)$ and $A \in \mathcal{B}$ with $\mu(A)>0, \lim _{\sup _{n \rightarrow \infty}} \frac{1}{N} \sum_{n=1}^{N} \mu(A \cap$ $\left.T^{-x_{n}} A\right)>0$.

Note that there are generalized polynomials $q(n)$ for which $\{q(n) \mid n \in \mathbb{Z}\}$ does not contain arbitrarily long polynomial progressions. For example, $\{[[\sqrt{2} n] \sqrt{2}] \mid$ $n \in \mathbb{Z}\}$ is shown not to be a set of recurrence in Section 4 .

Theorem 2.3. Let $q(n)$ be an integer-valued generalized polynomial. If $\{q(n) \mid$ $n \in \mathbb{N}\}$ contains arbitrarily long polynomial progressions $p_{m}(n), n=1, \ldots, N$, where

$$
p_{m}(x)=\sum_{i=1}^{l} a_{i}(m) x^{r_{i}}
$$

and where $r_{1}<r_{2}<\ldots<r_{l}$ are natural numbers and $a_{1}(n), \ldots, a_{l}(n)$ are generalized polynomials, then $\{q(n) \mid n \in \mathbb{N}\}$ is a set of recurrence. If there exists an increasing sequence $\left\{N_{i}\right\}_{i=1}^{\infty}$ such that the set

$$
S_{k}=S_{k}(q)=\left\{m \in \mathbb{N} \mid q(m n)=p_{m}(n), n=1,2, \ldots, N_{k}\right\}
$$

has positive upper density for any $k \in \mathbb{N}$ then $\{q(n) \mid n \in \mathbb{N}\}$ is an almost averaging set of recurrence. If in addition, the limit $\lim _{N \rightarrow \infty} \frac{1}{N} \sum_{n=1}^{N} e^{2 \pi i q(n) \lambda}$ exists for each $\lambda \in \mathbb{R}$, then $\{q(n) \mid n \in \mathbb{N}\}$ is an averaging set of recurrence.

Proof. The first part follows from Corollary 2.2. Let $(X, \mathcal{B}, \mu, T)$ be a measure preserving system and $A \in \mathcal{B}$ with $\mu(A)>0$. Let $R=\left\{\left(n^{r_{1}}, \ldots, n^{r_{l}}\right) \mid n \in \mathbb{N}\right\}$. By Proposition 2.1 there exist some $C(a) \in \mathbb{N}$ and $c(a)>0$ such that for each $n \in S_{k}$, where $k$ is chosen so that $N_{k}>C(a)$, there is some $n \in\left\{1,2, \ldots, N_{k}\right\}$ such that with $T_{i}=T^{a_{i}(m)}$, we have $\mu\left(A \cap T^{-p_{m}(n)} A\right)=\mu\left(A \cap T_{1}^{-n^{r_{1}}} \cdots T_{l}^{-n^{r_{l}}} A\right)>c(a)$. Clearly,

$$
\limsup _{N \rightarrow \infty} \frac{1}{N} \sum_{n=1}^{N} \mu\left(A \cap T^{-q(n)} A\right) \geq \limsup _{N \rightarrow \infty} \frac{1}{N} \sum_{m=1}^{[N / n]} \mu\left(A \cap T^{-q(m n)} A\right)
$$


for all $n=1,2, \ldots$, so that

$$
\begin{aligned}
\limsup _{N \rightarrow \infty} \frac{1}{N} \sum_{n=1}^{N} \mu\left(A \cap T^{-q(n)} A\right) & \geq \frac{1}{N_{k}} \sum_{n=1}^{N_{k}} \limsup _{N \rightarrow \infty} \frac{1}{N} \sum_{m=1}^{[N / n]} \mu\left(A \cap T^{-q(m n)} A\right) \\
& \geq \frac{1}{N_{k}} \limsup _{N \rightarrow \infty} \frac{1}{N} \sum_{m=1}^{\left[N / N_{k}\right]} \sum_{n=1}^{N_{k}} \mu\left(A \cap T^{-q(m n)} A\right) \\
& \geq \frac{1}{N_{k}} \limsup _{N \rightarrow \infty} \frac{1}{N} \sum_{m \in S_{k}\left(\left[N / N_{k}\right]\right)} \sum_{n=1}^{N_{k}} \mu\left(A \cap T^{-p_{m}(n)} A\right) \\
& >\frac{c(a)}{N_{k}^{2}} \bar{d}\left(S_{k}\right)>0,
\end{aligned}
$$

where $S_{k}\left(\left[N / N_{k}\right]\right)=S_{k} \cap\left\{1,2, \ldots,\left[N / N_{k}\right]\right\}$. By the observation made after Conjecture at the end of Section 1, we are done.

If

$$
q_{1}(n)=\left[\left[[\alpha n] \beta n^{2}\right]^{3}[\lambda n+\delta] \gamma n\right]
$$

is (a representation) of an integer-valued generalized polynomial, then $\alpha, \beta, \lambda, \delta, \gamma$ are called the coefficients of (the representation of) the generalized polynomial $q_{1}(n)$. Denote by $C(q)$ all the coefficients of an integer-valued generalized polynomial $q(n)$, and let $P(q)=\bigcup C(v)$, where the union is taken over all generalized polynomials $v(n)$ which are obtained from $q(n)$ by removing any number of nested brackets in $q(n)$. Thus $P\left(q_{1}\right)=C\left(q_{1}\right) \cup\{\alpha \beta, \alpha \beta \gamma, \beta \gamma, \lambda \gamma, \delta \gamma\}$.

Definition 2.4. A (representation of an) integer-valued generalized polynomial $q(n)$ has independent coefficients if $P(q) \cup\{1\}$ is rationally independent.

Proposition 2.5. If $q(n)$ is an integer-valued generalized polynomial with independent coefficients such that $q(0)=0$, then $\{q(n) \mid n \in \mathbb{N}\}$ is an almost averaging set of recurrence.

Proof. First, consider simple generalized polynomials $q(n)$ by which we mean generalized polynomials whose representations do not contain any sums, as for example

$$
q_{2}(n)=\left[[\alpha n] \beta n^{2}\right]^{3}[\lambda n] n .
$$

Note that $q_{1}(n)$ above is not simple. Since $q_{2}(n)$ has independent coefficients, then $\left(\alpha n,[\alpha n] \beta n^{2}, \lambda n\right), n=1,2, \ldots$, is uniformly distributed $(\bmod 1)$ in $\mathbb{R}^{3},[10$, Prop. 3.5]. By (1), $[x m]=m[x]$ iff $\{x\}<1 / m$. So if $m$ is in the set

$$
\left\{m \in \mathbb{N} \mid\{\alpha m\}<\frac{1}{k},\left\{[\alpha m] \beta m^{2}\right\}<\frac{1}{k^{3}},\{\lambda m\}<\frac{1}{k}\right\}
$$

which has positive density, then

$$
\left[[\alpha m n] \beta(m n)^{2}\right]^{3}[\lambda m n] m n=\left[[\alpha m] \beta m^{2} n^{3}\right]^{3}[\lambda m] m n^{2}=\left[[\alpha m] \beta m^{2}\right]^{3}[\lambda m] m n^{11}
$$


for $n=1,2, \ldots, k$. Hence, $S_{k}\left(q_{2}\right)$ defined by (2) (with $N_{k}=k$ ) is contained in the set (3), and it follows from Theorem 2.3 that $\left\{q_{2}(n) \mid n \in \mathbb{N}\right\}$ is an almost averaging set of recurrence. We call $\alpha n,[\alpha n] \beta n^{2}, \lambda n$ the (inner) subpolynomials of $q_{2}(n)$. The proof for any simple generalized polynomial $q(n)$ is done the same way. For if $v_{1}(n), \ldots, v_{l}(n)$ are all the subpolynomials of $q(n)$, then by [10, Prop. 3.5], $\left(v_{1}(n), \ldots, v_{l}(n)\right), n=1,2, \ldots$, is uniformly distributed $(\bmod 1)$ in $\mathbb{R}^{l}$ so that the fractional part of each $v_{i}(n)$ can be chosen arbitrary small.

Now if $q(n)$ is not simple, then by [10, Lemma 2.7], we have

$$
q(n)=\sum_{i=1}^{L} q_{i}(n)+\sum_{i} t_{i}(n) 1_{A}\left(r_{1 i}(n), r_{2 i}(n)\right)
$$

where $1_{A}(x, y)=\left\{\begin{array}{lll}1 & \text { if } & \{x\}+\{y\} \geq 1 \\ 0 & \text { if } & \{x\}+\{y\}<1\end{array}\right.$ and where each $q_{i}(n)$ is a simple nonconstant generalized polynomial and $r_{j i}(n)$ is a sum of simple subpolynomials of $q_{i}(n)$ (possibly with some indicator functions involved). Let $N_{k}$ be given and let $v_{1}(n), \ldots, v_{l}(n)$ be all the subpolynomials involved in the $q_{i}(n)$ 's and $r_{j i}(n)$ 's. Then by [10, Prop. 3.5], $\left(v_{1}(n), \ldots, v_{l}(n)\right), n=1,2, \ldots$, is uniformly distributed $(\bmod 1)$ so that the fractional part of each $v_{i}(m)$ can be chosen small enough to make each $1_{A}\left(r_{1 i}(m n), r_{2 i}(m n)\right)=0$ for $n=1, \ldots, N_{k}$. For these $m$ 's, we have $q(m n)=\sum_{i=1}^{L} q_{i}(m n)$, and similarly as in the above simple case, $q(m n)=$ $\sum_{i=1}^{L} q_{i}(m) n^{\operatorname{deg}\left(q_{i}\right)}$ for $n=1, \ldots, N_{k}$.

This method of finding arbitrarily long polynomial progressions in $\{[q(n)] \mid n \in$ $\mathbb{N}\}$, works for many other generalized polynomials as well. For example,

$$
\left\{\left[\alpha_{1} n\right]\left[\alpha_{2} n\right] \cdots\left[\alpha_{k} n\right] \mid n \in \mathbb{N}\right\}
$$

is an averaging set of recurrence for any $k \in \mathbb{N}, \alpha_{i} \in \mathbb{R}$, since for any $N \in \mathbb{N}$, there exists $k_{1} \in \mathbb{N}, 1<k_{1} \leq k$, such that by possibly reordering the $\alpha_{i}$ 's the set $M$ of $m \in \mathbb{N}$ for which $\left\{\alpha_{i} m\right\}<1 / N, i=1, \ldots, k_{1}$ and $\left\{\alpha_{i} m\right\}>1-1 / N$, $i=k_{1}+1, \ldots, k$, has positive density. So for $m \in M$ and $n=1,2, \ldots, N$ we have $\left[\alpha_{i} m n\right]=n\left[\alpha_{i} m\right], i=1, \ldots, k_{1}$ and $\left[\alpha_{i} m n\right]=n\left[\alpha_{i} m\right]+n-1, i=k_{1}+1, \ldots, k$ so that $\left[\alpha_{1} m n\right]\left[\alpha_{2} m n\right] \cdots\left[\alpha_{k} m n\right]=n^{k}\left[\alpha_{1} m\right]\left[\alpha_{2} m\right] \cdots\left[\alpha_{k} m\right]+p_{m}(n), n=1,2, \ldots, N$, where $p_{m}(n)$ is a polynomial without a constant term. Furthermore,

$$
\lim _{N \rightarrow \infty} \frac{1}{N} \sum_{n=1}^{N} e^{2 \pi i\left[\alpha_{1} n\right]\left[\alpha_{2} n\right] \cdots\left[\alpha_{k} n\right] \lambda}
$$

exists for any $\lambda \in \mathbb{R}$. For by [11], if $\left[\alpha_{1} n\right]\left[\alpha_{2} n\right] \cdots\left[\alpha_{k} n\right] \lambda, n=1,2, \ldots$, is not uniformly distributed $(\bmod 1)$, then either $\lambda$ is rational or $k<3$ and $\lambda$ depends rationally on $1,1 / \alpha_{1}(k=1)$ or on $1, \alpha_{1} / \alpha_{2}=\sqrt{c}$ for some $c \in \mathbb{Q}^{+}(k=2)$, in which cases we have identities like $[\alpha n] \frac{1}{\alpha}=n-\{\alpha n\} \frac{1}{\alpha}$ and

$$
[\alpha \sqrt{c} n][\alpha n] \sqrt{c} \equiv-\frac{1}{2}(\{\alpha \sqrt{c}\}-\sqrt{c}\{\alpha n\})^{2} \quad(\bmod 1)
$$


If $1, \alpha, \alpha \beta$ are rationally independent, then the set $\{[[\alpha n] \beta] \mid n \in \mathbb{N}\}$ contains arbitrarily long progressions starting at 0 since $(\alpha n,[\alpha n] \beta), n=1,2, \ldots$, is uniformly distributed $(\bmod 1)$. However, if $\alpha \beta$ is rationally dependent on $1, \alpha$, then it is not obvious if one can find such progressions. We need therefore a different method. In Section 4 we show that $\left\{[[\sqrt{2} n] \sqrt{2}]^{2} \mid n \in \mathbb{N}\right\}$ is a set of recurrence while $\{[[\sqrt{2} n] \sqrt{2}] \mid n \in \mathbb{N}\}$ is not. Therefore, $\left\{[[\sqrt{2} n] \sqrt{2}]^{2} \mid n \in \mathbb{N}\right\}$ cannot contain any quadratic polynomial progressions, and possibly no polynomial progressions at all.

\section{The SPECTRAL METHOD}

Furstenberg used spectral theory in [7] to show that polynomials $p(t)$ taking integer values on the integers and with $p(0)=0$, form sets of recurrence. We shall use a similar method.

Recall that a real-valued sequence $y_{n}, n=1,2, \ldots$, is uniformly distributed $(\bmod 1)$ if and only if for any Riemann-integrable periodic $\bmod 1$ function $g$, $\frac{1}{N} \sum_{n=1}^{N} g\left(y_{n}\right) \rightarrow \int_{0}^{1} g d x$, and that this is equivalent to Weyl's criterion:

$$
\frac{1}{N} \sum_{n=1}^{N} e^{2 \pi i r y_{n}} \rightarrow 0 \quad \text { for all } \quad r \in \mathbb{Z} \backslash\{0\} .
$$

Let $\left\{x_{n} \mid n \in \mathbb{N}\right\}$ be a subset of $\mathbb{Z}$, and let

$$
\Lambda=\Lambda\left(x_{n}\right)=\left\{\lambda \in \mathbb{R} \mid x_{n} \lambda, n=1,2, \ldots, \text { is not uniformly distributed }(\bmod 1)\right\} .
$$

For many integer-valued generalized polynomials $q(n)$ we can show that the sequence $q(n) \lambda, n=1,2, \ldots$, is uniformly distributed $(\bmod 1)$ when $\lambda \in \mathbb{R}$ is outside a certain countably set $\Lambda_{e}$ which only depends on the coefficients of $q(n)$. For example,

$$
[[\alpha n] \beta] \lambda=\alpha \beta \lambda n-\{\alpha \beta n-\{\alpha n\} \beta\} \lambda, n=1,2, \ldots
$$

is uniformly distributed $(\bmod 1)$ if $\alpha \beta \lambda$ is rationally independent of $1, \alpha, \alpha \beta$, i.e. if $\lambda \notin \Lambda_{e}=\left\{a+\frac{b}{\beta}+\frac{c}{\alpha \beta} \mid a, b, c \in \mathbb{Q}\right\}$. Here it depends on $\alpha$ and $\beta$ if $\Lambda$ is equal to $\Lambda_{e}$.

Often it is hard to determine precisely what $\Lambda\left(x_{n}\right)$ is. Therefore, to avoid unnecessary problems of finding $\Lambda\left(x_{n}\right)$, we shall sometimes let $\Lambda_{e}=\Lambda_{e}\left(x_{n}\right)$ to be a subset of $\mathbb{R}$ containing $\Lambda\left(x_{n}\right)$. Note that $\mathbb{Q} \subseteq \Lambda\left(x_{n}\right) \subseteq \Lambda_{e}\left(x_{n}\right)$, so that $\Lambda\left(x_{n}\right)$ is always infinite. However, it is not known whether $\Lambda(q(n))$ is countable for any integer-valued generalized polynomial $q(n)$.

Definition 3.1. An integer valued sequence $x_{n}, n=1,2, \ldots$, has property $P$ if there exists a countable set $\Lambda_{e}$ containing $\Lambda\left(x_{n}\right)$ and such that for any $\lambda_{1}, \ldots, \lambda_{l} \in$ $\Lambda_{e}$ there exist real-valued sequences $v_{1}(n), \ldots, v_{k}(n), n=1,2, \ldots$, and Riemannintegrable periodic mod 1 functions $g_{i}$ on $\mathbb{R}^{k}$ with $x_{n} \lambda_{i}=g_{i}\left(v_{1}(n), \ldots, v_{k}(n)\right)$, $i=1,2, \ldots, l$, and such that

$$
\left(x_{n} \theta, v_{1}(n), \ldots, v_{k}(n)\right), \quad n=1,2, \ldots
$$

is uniformly distributed $(\bmod 1)$ in $\mathbb{R}^{k+1}$ for any $\theta \notin \Lambda_{e}$. 
Question. Is it true that for any integer-valued generalized polynomial $q(n)$, the sequence $q(n), n=1,2, \ldots$, has property $P$ ?

Let $\left\{x_{n} \mid n \in \mathbb{N}\right\} \subset \mathbb{Z}$ be a sequence having the property $\mathrm{P}$ and let $\Lambda_{e}\left(x_{n}\right) \supseteq$ $\Lambda\left(x_{n}\right)$ be a countable set. If $(X, \mathcal{B}, \mu, T)$ is a measure preserving system, then denote by $\mathcal{H}_{\Lambda_{e}}$ the subspace of $\mathcal{H}=L^{2}(X, \mathcal{B}, \mu)$ spanned by the eigenfunctions of $T$ corresponding to the eigenvalues $e^{2 \pi i \lambda}, \lambda \in \Lambda_{e}\left(x_{n}\right)$. If $\delta>0$ and $\Lambda_{0} \subset \Lambda_{e}\left(x_{n}\right)$ is a finite subset, let

$$
G=G\left(\Lambda_{0}, \delta\right)=\left\{n \in \mathbb{N}|| e^{2 \pi i x_{n} \lambda}-1 \mid<\delta \text { for all } \lambda \in \Lambda_{0}\right\}
$$

and let $G_{N}=G \cap\{1,2, \ldots, N\}$.

Lemma 3.2. If $x_{n}, n=1,2, \ldots$, is an integer-valued sequence with the property $P$, let $\Lambda_{e}=\Lambda_{e}\left(x_{n}\right) \supseteq \Lambda\left(x_{n}\right)$ be a countable set, let $\Lambda_{0}$ be some finite subset of $\Lambda_{e}$, let $\delta>0$ and let $G=G\left(\Lambda_{0}, \delta\right)$. Then $d(G)=\lim _{N \rightarrow \infty} \frac{\left|G_{N}\right|}{N}$ exists, and for any $\lambda \in \mathbb{R}$, the limits

$$
\lim _{N \rightarrow \infty} \frac{1}{\left|G_{N}\right|} \sum_{n \in G_{N}} e^{2 \pi i x_{n} \lambda} \quad \text { and } \quad \lim _{N \rightarrow \infty} \frac{1}{N} \sum_{n=1}^{N} e^{2 \pi i x_{n} \lambda}
$$

exist. If $\lambda \notin \Lambda_{e}$, then both limits in (5) equal 0 .

Proof. Let $\Lambda_{0}=\left\{\lambda_{1}, \ldots, \lambda_{l}\right\}$. Since $x_{n}, n=1,2, \ldots$, has the property P, there exist Riemann-integrable periodic $\bmod 1$ functions $g_{i}, i=1, \ldots, l$, and sequences $v_{1}(n), \ldots, v_{k}(n), n=1,2, \ldots$, such that $\left(v_{1}(n), \ldots, v_{k}(n)\right)$ is uniformly distributed $(\bmod 1)$ and $x_{n} \lambda_{i}=g_{i}\left(v_{1}(n), \ldots, v_{k}(n)\right)$ for all $\lambda_{i} \in \Lambda_{0}$. Let $\varepsilon>0$ be such that if $\{y\}=y-[y] \in I(\varepsilon)=[0, \varepsilon) \cup(1-\varepsilon, 1]$ then $\left|e^{2 \pi i y}-1\right|<\delta$. Define

$$
g_{0}\left(y_{1}, \ldots, y_{k}\right)=\prod_{i=1}^{l} 1_{I(\varepsilon)}\left(\left\{g_{i}\left(y_{1}, \ldots, y_{k}\right)\right\}\right) .
$$

Then $n \in G$ if and only if $g_{0}\left(v_{1}(n), \ldots, v_{k}(n)\right)=\prod_{i=1}^{l} 1_{I(\varepsilon)}\left(\left\{x_{n} \lambda_{i}\right\}\right)=1$. Therefore,

$$
d(G)=\lim _{N \rightarrow \infty} \frac{\left|G_{N}\right|}{N}=\lim _{N \rightarrow \infty} \frac{1}{N} \sum_{n=1}^{N} g_{0}\left(v_{1}(n), \ldots, v_{k}(n)\right)=\int_{0}^{1} \ldots \int_{0}^{1} g_{0} d y_{1} \cdots d y_{k}
$$

exists.

If $\lambda \notin \Lambda_{e}$, then by the definition of $\Lambda_{e}, \lim _{N \rightarrow \infty} \frac{1}{N} \sum_{n=1}^{N} e^{2 \pi i x_{n} \lambda}=0$, and

$$
\begin{aligned}
\frac{1}{\left|G_{N}\right|} \sum_{n \in G_{N}} e^{2 \pi i x_{n} \lambda} & =\frac{1}{\left|G_{N}\right|} \sum_{n=1}^{N} e^{2 \pi i x_{n} \lambda} g_{0}\left(v_{1}(n), \ldots, v_{k}(n)\right) \\
& =\frac{N}{\left|G_{N}\right|} \frac{1}{N} \sum_{n=1}^{N} g\left(x_{n} \lambda, v_{1}(n), \ldots, v_{k}(n)\right)
\end{aligned}
$$


where $g\left(y_{0}, y_{1}, \ldots, y_{k}\right)=e^{2 \pi i y_{0}} g_{0}\left(y_{1}, \ldots, y_{k}\right)$ is a Riemann-integrable periodic mod 1 function. Since $\left(x_{n} \lambda, v_{1}(n), \ldots, v_{k}(n)\right), n=1,2, \ldots$, is uniformly distributed $(\bmod 1),(6)$ converges to $\frac{1}{d(G)} \int_{0}^{1} \cdots \int_{0}^{1} g d y_{0} \cdots d y_{k}=0$.

If $\lambda \in \Lambda_{e}$, then for $\lambda, \lambda_{1}, \ldots, \lambda_{l}$ there exist Riemann-integrable periodic mod 1 functions $f$ and $g_{i}, i=1, \ldots, l$, and real-valued sequences $v_{1}(n), \ldots, v_{k}(n), n=$ $1,2, \ldots$, so that $x_{n} \lambda=f\left(v_{1}(n), \ldots, v_{k}(n)\right)$ and $x_{n} \lambda_{i}=g_{i}\left(v_{1}(n), \ldots, v_{k}(n)\right)$ for all $\lambda_{i} \in \Lambda_{0}$. So

$$
\begin{aligned}
\lim _{N \rightarrow \infty} \frac{1}{N} \sum_{n=1}^{N} e^{2 \pi i x_{n} \lambda} & =\lim _{N \rightarrow \infty} \frac{1}{N} \sum_{n=1}^{N} e^{2 \pi i f\left(v_{1}(n), \ldots, v_{k}(n)\right)} \\
& =\int_{0}^{1} \cdots \int_{0}^{1} e^{2 \pi i f\left(y_{1}, \ldots, y_{k}\right)} d y_{1} \cdots d y_{k}
\end{aligned}
$$

and

$$
\begin{aligned}
\lim _{N \rightarrow \infty} \frac{1}{\left|G_{N}\right|} \sum_{n \in G_{N}} e^{2 \pi i x_{n} \lambda} & =\lim _{N \rightarrow \infty} \frac{1}{\left|G_{N}\right|} \sum_{n=1}^{N} e^{2 \pi i f\left(v_{1}(n), \ldots, v_{k}(n)\right)} g\left(v_{1}(n), \ldots, v_{k}(n)\right) \\
& =\frac{1}{d(G)} \int_{0}^{1} \cdots \int_{0}^{1} e^{2 \pi i f\left(y_{1}, \ldots, y_{k}\right)} g\left(y_{1}, \ldots, y_{k}\right) d y_{1} \cdots d y_{k}
\end{aligned}
$$

Lemma 3.3. Let $x_{n}, n=1,2, \ldots$, be an integer-valued sequence with property $P$, let $\Lambda_{e}=\Lambda_{e}\left(x_{n}\right) \supseteq \Lambda\left(x_{n}\right)$ be a countable set, let $\Lambda_{0}$ be some finite subset of $\Lambda_{e}$, let $\delta>0$ and let $G=G\left(\Lambda_{0}, \delta\right)$ be defined by (4). If $f$ is orthogonal to any function in $\mathcal{H}_{\Lambda_{e}}$, then

$$
\lim _{N \rightarrow \infty} \frac{1}{\left|G_{N}\right|} \sum_{n \in G_{N}} T^{x_{n}} f=0 .
$$

Proof. By the spectral theorem,

$$
\begin{aligned}
\left\|\frac{1}{\left|G_{N}\right|} \sum_{n \in G_{N}} T^{x_{n}} f\right\|^{2} & =\frac{1}{\left|G_{N}\right|^{2}} \sum_{n, m \in G_{N}}\left\langle T^{x_{n}-x_{m}} f, f\right\rangle \\
& =\frac{1}{\left|G_{N}\right|^{2}} \sum_{n, m \in G_{N}} \int_{0}^{1} e^{2 \pi i\left(x_{n}-x_{m}\right) \lambda} d \nu_{f}(\lambda) \\
& =\int_{0}^{1}\left|\frac{1}{\left|G_{N}\right|} \sum_{n \in G_{N}} e^{2 \pi i x_{n} \lambda}\right|^{2} d \nu_{f}(\lambda) .
\end{aligned}
$$

By Lemma 3.2, $\frac{1}{\left|G_{N}\right|} \sum_{n \in G_{N}} e^{2 \pi i x_{n} \lambda} \rightarrow 0$ for all $\lambda \notin \Lambda_{e}$. Since $f$ is orthogonal to $\mathcal{H}_{\Lambda_{e}}$, the measure $\nu_{f}$ does not have atoms in $\Lambda_{e}$. Therefore, since $\Lambda_{e}$ is countable, we have

$$
\frac{1}{\left|G_{N}\right|} \sum_{n \in G_{N}} e^{2 \pi i x_{n} \lambda} \rightarrow 0 \quad \text { a.e. }\left(\nu_{f}\right) \text {. }
$$

Hence, (7) converges to 0. 
Theorem 3.4. Let $x_{n}, n=1,2, \ldots$, be an integer-valued sequence with property $P$, and let $\Lambda_{e}=\Lambda_{e}\left(x_{n}\right) \supseteq \Lambda\left(x_{n}\right)$ be a countable set. If for any $\delta>0$ and any finite subset $\Lambda_{0} \subset \Lambda_{e}$, the set $G\left(\Lambda_{0}, \delta\right)$ defined by (4) has positive density, then the set $\left\{x_{n} \mid n \in \mathbb{N}\right\}$ is an averaging set of recurrence. On the other hand, if for some $\Lambda_{0} \subset \Lambda_{e}$ and $\varepsilon>0$, the set $G\left(\Lambda_{0}, \varepsilon\right)$ is of zero density, then $\left\{x_{n} \mid n \in \mathbb{N}\right\}$ cannot be an averaging set of recurrence, and if for some $\Lambda_{0} \subset \Lambda_{e}$ and $\varepsilon>0$, the set $G\left(\Lambda_{0}, \varepsilon\right)$ is finite, then $\left\{x_{n} \mid n \in \mathbb{N}\right\}$ is not a set of recurrence.

Question. Is there an example of an integer-valued sequence $x_{n}, n=1,2, \ldots$, for which $\left\{x_{n} \mid n \in \mathbb{N}\right\}$ is a set recurrence and such that there exist $\Lambda_{0} \subset \Lambda_{e}\left(x_{n}\right)$ and $\varepsilon>0$ so that the set $G\left(\Lambda_{0}, \varepsilon\right)$ is of zero density?

Proof. Let $(X, \mathcal{B}, \mu, T)$ be a measure preserving system and let $A \in \mathcal{B}$ with $\mu(A)>$ 0 . We will show that if all $G\left(\Lambda_{0}, \delta\right)$ has positive density, then

$$
\lim _{N \rightarrow \infty} \frac{1}{N} \sum_{n=1}^{N} \mu\left(A \cap T^{-x_{n}} A\right)=\lim _{N \rightarrow \infty} \frac{1}{N} \sum_{n=1}^{N}\left\langle 1_{A}, T^{x_{n}} 1_{A}\right\rangle>0 .
$$

As before, denote by $\mathcal{H}_{\Lambda_{e}}$ the subspace of $\mathcal{H}=L^{2}(X, \mathcal{B}, \mu)$ spanned by the eigenfunctions of $T$ corresponding to the eigenvalues $e^{2 \pi i \lambda}, \lambda \in \Lambda_{e}\left(x_{n}\right)$. Let $f \in \mathcal{H}$ be such that

$$
\lim _{N \rightarrow \infty} \frac{1}{\left|G_{N}\right|} \sum_{n \in G_{N}}\left\langle f, T^{x_{n}} f\right\rangle=0
$$

for any $G=G\left(\Lambda_{0}, \delta\right)$. We have $f=f_{1}+f_{2}$, where $f_{1} \in \mathcal{H}_{\Lambda_{e}}$ and $f_{2}$ is orthogonal to $\mathcal{H}_{\Lambda_{e}}$. Since $f_{1} \in \mathcal{H}_{\Lambda_{e}}$, we have $f_{1}=\sum_{\lambda \in \Lambda_{e}} a_{\lambda} g_{\lambda}$, where $T g_{\lambda}=e^{2 \pi i \lambda} g_{\lambda}$ and $\left|g_{\lambda}\right|=1$. Let $\varepsilon>0$, and choose a finite subset $\Lambda_{0} \subset \Lambda_{e}$ so that $\sum_{\lambda \notin \Lambda_{0}}\left|a_{\lambda}\right|^{2}<\varepsilon$.

Then

$$
\left\|f_{1}-\sum_{\lambda \in \Lambda_{0}} a_{\lambda} g_{\lambda}\right\|=\left\|\sum_{\lambda \notin \Lambda_{0}} a_{\lambda} g_{\lambda}\right\|<\varepsilon .
$$

Let $0<\delta<\frac{\varepsilon}{\left\|f_{1}\right\|}$ and $G=G\left(\Lambda_{0}, \delta\right)$. By assumption, $d(G)>0$. If $n \in G$, then

$$
\begin{aligned}
& \left\|f_{1}-T^{x_{n}} f_{1}\right\| \\
& \leq\left\|f_{1}-\sum_{\lambda \in \Lambda_{0}} a_{\lambda} g_{\lambda}\right\|+\left\|\sum_{\lambda \in \Lambda_{0}} a_{\lambda} g_{\lambda}-T^{x_{n}} \sum_{\lambda \in \Lambda_{0}} a_{\lambda} g_{\lambda}\right\|+\left\|T^{x_{n}} \sum_{\lambda \in \Lambda_{0}} a_{\lambda} g_{\lambda}-T^{x_{n}} f_{1}\right\| \\
& \leq 2 \varepsilon+\left\|\sum_{\lambda \in \Lambda_{0}} a_{\lambda}\left(1-e^{2 \pi i x_{n} \lambda}\right) g_{\lambda}\right\| \leq 2 \varepsilon+\delta\left\|f_{1}\right\|<3 \varepsilon,
\end{aligned}
$$

so that

$$
\left\|\frac{1}{\left|G_{N}\right|} \sum_{n \in G_{N}} T^{x_{n}} f_{1}-f_{1}\right\|<3 \varepsilon .
$$

By Lemma $3.3, \frac{1}{\left|G_{N}\right|} \sum_{n \in G_{N}} T^{x_{n}} f_{2} \rightarrow 0$. So for large $N$,

$$
\left\|\frac{1}{\left|G_{N}\right|} \sum_{n \in G_{N}} T^{x_{n}} f-f_{1}\right\|<3 \varepsilon .
$$


Since $\left|\left\langle\frac{1}{\left|G_{N}\right|} \sum_{n \in G_{N}} T^{x_{n}} f-f_{1}, f\right\rangle\right| \leq 3 \varepsilon\|f\|$ and

$$
\left|\left\langle\frac{1}{\left|G_{N}\right|} \sum_{n \in G_{N}} T^{x_{n}} f-f_{1}, f\right\rangle\right|=\left|\frac{1}{\left|G_{N}\right|} \sum_{n \in G_{N}}\left\langle T^{x_{n}} f, f\right\rangle-\left\langle f_{1}, f\right\rangle\right| \rightarrow\left|\left\langle f_{1}, f\right\rangle\right|
$$

for any $\varepsilon>0$, it follows that $\left\langle f_{1}, f\right\rangle=0$, i.e. $f$ is orthogonal to $\mathcal{H}_{\Lambda_{e}}$.

Consider now $f=1_{A}$. Since $\left\langle 1_{A}, 1\right\rangle=\mu(A)>0$ and $1 \in \mathcal{H}_{\Lambda_{e}}, 1_{A}$ is not orthogonal to $\mathcal{H}_{\Lambda_{e}}$. Hence,

$$
\limsup _{N \rightarrow \infty} \frac{1}{\left|G_{N}\right|} \sum_{n \in G_{N}} \mu\left(A \cap T^{-x_{n}} A\right)=a>0
$$

for some $G=G\left(\Lambda_{0}, \delta\right)$ of positive density. By Lemma 3.2, $\lim _{N \rightarrow \infty} \frac{1}{\left|G_{N}\right|} \sum_{n \in G_{N}} e^{2 \pi i x_{n} \lambda}$ exists for any $\lambda \in \mathbb{R}$. So by the comments at the end of Section 1

$$
\lim _{N \rightarrow \infty} \frac{1}{\left|G_{N}\right|} \sum_{n \in G_{N}} \mu\left(A \cap T^{-x_{n}} A\right)
$$

exists. We therefore get

$$
\lim _{N \rightarrow \infty} \frac{1}{N} \sum_{n=1}^{N} \mu\left(A \cap T^{-x_{n}} A\right) \geq \lim _{N \rightarrow \infty} \frac{\left|G_{N}\right|}{N} \frac{1}{\left|G_{N}\right|} \sum_{n \in G_{N}} \mu\left(A \cap T^{-x_{n}} A\right)=d(G) a>0
$$

which shows that $\left\{x_{n} \mid n \in \mathbb{N}\right\}$ is an averaging set of recurrence.

Now, let $\Lambda_{0}=\left\{\lambda_{1}, \ldots, \lambda_{l}\right\}$ and $\delta>0$, and consider the measure preserving system $\left(K^{l}, \mathcal{B}, \mu, T\right)$, where $K^{l} \simeq \mathbb{R}^{l} / \mathbb{Z}^{l}$ is the $l$-dimensional torus and

$$
T\left(x_{1}, x_{2}, \ldots, x_{l}\right)=\left(x_{1}+\lambda_{1}, x_{2}+\lambda_{2}, \ldots, x_{l}+\lambda_{l}\right) .
$$

Let

$$
A=\left\{y \in K^{l}|| y_{i}-1 \mid<\delta / 2, i=1,2, \ldots, l\right\} .
$$

For each $n$ for which $\mu\left(A \cap T^{-x_{n}} A\right)>0$, there exists $\left(e^{2 \pi i z_{1}}, \ldots, e^{2 \pi i z_{l}}\right) \in A \cap T^{-x_{n}} A$ so that $\left|e^{2 \pi i z_{i}}-1\right|<\delta / 2$ and $\left|e^{2 \pi i\left(z_{i}+x_{n} \lambda_{i}\right)}-1\right|<\delta / 2$ for $i=1,2, \ldots, l$. This implies that $\left|e^{2 \pi i x_{n} \lambda_{i}}-1\right|<\delta$ for $i=1,2, \ldots, l$. Hence,

$$
\left\{n \mid \mu\left(A \cap T^{-x_{n}} A\right)>0\right\} \subset G\left(\Lambda_{0}, \delta\right)
$$

which shows that $\left\{x_{n} \mid n \in \mathbb{N}\right\}$ cannot be an averaging set of recurrence if $G\left(\Lambda_{0}, \delta\right)$ has zero density. Furthermore, if $G\left(\Lambda_{0}, \delta\right)=\left\{n_{1}, \ldots, n_{r}\right\}$ then let $\Lambda_{1}=$ $\left\{\lambda_{1}, \ldots, \lambda_{l}, \theta_{1}, \ldots, \theta_{r}\right\}$ where $\theta_{i}=\theta_{0} / x_{n_{i}}$ and $\theta_{0}$ is chosen so that $\left|e^{2 \pi i \theta_{0}}-1\right|>\delta$. This way $\left|e^{2 \pi i x_{n_{i}} \theta_{i}}-1\right|>\delta$ for each $i=1, \ldots, r$, so that $G\left(\Lambda_{1}, \delta\right)=\emptyset$. Hence, by using ( eq-sub) with $\Lambda_{1}$ instead of $\Lambda_{0}$ and for a new system $\left(K^{l+r}, \mathcal{B}, \mu, T\right)$, we see that $\left\{x_{n} \mid n \in \mathbb{N}\right\}$ is not a set of recurrence. 
We shall apply this theorem to what we call manageable generalized polynomials. If

$$
q(n)=\left[\left[\alpha n^{2}+\beta n\right] \lambda n\right][\gamma n]
$$

then we call $\alpha n^{2}, \beta n, \lambda n, \gamma n,\left[\alpha n^{2}\right] \lambda n,[\beta n] \lambda n,\left[\alpha n^{2}+\beta n\right] \lambda n$ subpolynomials of $q(n)$. We say that $q(n)$ is manageable if for any subpolynomials $a_{1}(n), \ldots, a_{k}(n)$ of $q(n)$ there exist generalized polynomials $u_{1}(n), \ldots, u_{l}(n)$ and Riemann-integrable periodic mod 1 functions $g_{i}$ on $\mathbb{R}^{l}, i=1, \ldots, k$, such that $\left(u_{1}(n), \ldots, u_{l}(n)\right), n=$ $1,2, \ldots$, is uniformly distributed $(\bmod 1)$ and such that

$$
a_{i}(n)=g_{i}\left(u_{1}(n), \ldots, u_{l}(n)\right), i=1, \ldots, k .
$$

Note that if all generalized polynomials are manageable, then the answer to the question at the end of Section 1 is positive.

Corollary 3.5. If $q(n)$ is a manageable integer-valued generalized polynomial and $q(n) \lambda, n=1,2, \ldots$, is uniformly distributed $(\bmod 1)$ for any irrational number $\lambda$, then $\{q(n) \mid n \in \mathbb{N}\}$ is an averaging set of recurrence if $d(\{n \in \mathbb{N} \mid q(n) \equiv 0$ $(\bmod s)\})>0$ for each $s \in \mathbb{N}$.

Proof. Here we let $\Lambda_{e}=\Lambda=\mathbb{Q}$. So since $q(n)$ is manageable, $q(n)$ has the property $\mathrm{P}$ and $d(\{n \in \mathbb{N} \mid q(n) \equiv 0(\bmod s)\})$ exists for each $s \in \mathbb{N}$. If $\Lambda_{0}=\left\{\frac{r_{1}}{s_{1}}, \ldots, \frac{r_{l}}{s_{l}}\right\}$ and $\delta<\min \left\{\frac{1}{s_{i}} \mid i=1, \ldots, l\right\}$, then

$$
\begin{aligned}
G\left(\Lambda_{0}, \delta\right) & =\left\{n \in \mathbb{N} \mid q(n) \frac{r_{i}}{s_{i}} \equiv 0(\bmod 1), i=1, \ldots, l\right\} \\
& =\left\{n \in \mathbb{N} \mid q(n) \equiv 0(\bmod s), s \text { is least common multiple of } s_{1}, \ldots, s_{l}\right\} .
\end{aligned}
$$

For example, the generalized polynomials $\left[\alpha_{1} n\right]\left[\alpha_{2} n\right] \cdots\left[\alpha_{k} n\right], k \geq 3$, and $[\alpha n] n^{2}$ satisfy the conditions of Corollary 3.5 (see [11] and [9, Thm. 3.2.5]). However, there are many generalized polynomials which this corollary does not apply to. For example, if $q(n)=\left[\left[q_{0}(n)\right] \beta n^{l}\right]$ then $q(n) \lambda=\left[q_{0}(n)\right] \beta \lambda n^{l}-\left\{\left[q_{0}(n)\right] \beta n^{l}\right\} \lambda$ is not uniformly distributed $(\bmod 1)$ if $\lambda=1 / \beta$. The next proposition covers far more generalized polynomials.

We shall use a result from [8] on IP-recurrent functions. A subset $S \subset \mathbb{N}$ is called an IP-set if it consists of a sequence $p_{1}, p_{2}, \ldots$ together with all finite sums $p_{i_{1}}+p_{i_{2}}+\cdots+p_{i_{k}}$ with $i_{1}<i_{2}<\cdots<i_{k}$. A bounded function $f(n)$ is called IP-recurrent if for any $\varepsilon>0$ the set

$$
\{n \in \mathbb{N}|| f(n)-f(0) \mid<\varepsilon\}
$$

has non-empty intersection with any IP-set. A set which non-trivially intersects any IP-set is called an IP*-set. It is known that the intersection of finitely many $\mathrm{IP}^{*}$-sets is an IP*-set. Any IP*-set has bounded gaps and hence has positive upper density. (See [7] for more details.) So if $f_{1}(n), \ldots, f_{k}(n)$ are IP-recurrent, then for any $\varepsilon>0$ the set

$$
\left\{n \in \mathbb{N}|| f_{i}(n)-f_{i}(0) \mid<\varepsilon, i=1,2, \ldots, k\right\}
$$

is an IP*-set, and in particular it has positive upper density. A function $g(n)$ is called LIPR if $e^{2 \pi i g(n) \lambda}$ is IP-recurrent for any $\lambda \in \mathbb{R}$. 
Lemma 3.6 [8]. Let $q(n)$ be a generalized polynomial. There exists a countable set $\Theta(q)$ such that if $\theta \in \mathbb{R} \backslash \Theta(q)$, and each bracket in $q(n)$ is replaced by the function $I_{\theta}(x)=[x+\theta]$, then the resulting generalized polynomial $q_{\theta}(n)$ is LIPR.

So if $q(n)=[[\alpha n] \beta n]$, then $q_{\theta}(n)=[[\alpha n+\theta] \beta n+\theta]$ for some $\theta \in \mathbb{R}$. For example, $[\alpha n+\theta]^{k}$ are LIPR if and only if $\theta \neq l \alpha, l \in \mathbb{Z}$, [9, Prop. 4.2.5].

Remark. It is not necessary to use the same $\theta$ for all the brackets even though we have found it convenient to do so here.

Proposition 3.7. Let $q(n)$ be a manageable integer-valued generalized polynomial which has the property $P$ and is such that $q(0)=0$. Then there exists a real number $\theta$ such that if $q_{\theta}(n)$ is the generalized polynomial obtained from $q(n)$ by replacing each bracket by $I_{\theta}$, the set

$$
\left\{q_{\theta}(n) \mid n \in \mathbb{N}\right\}
$$

is an averaging set of recurrence.

Proof. By Lemma 3.6, the $\theta$ 's may be chosen arbitrary small so that $q_{\theta}(0)=0$. Since $q(n)$ is manageable it follows that if $q(n) \lambda$ is uniformly distributed $(\bmod 1)$, then $q_{\theta}(n) \lambda$ is also uniformly distributed $(\bmod 1)$, and $q_{\theta}(n)$ inherits the property P. The proposition now follows by Lemma 3.6 and Theorem 3.4.

Note that there are many generalized polynomials $q(n)$ for which $\{q(n) \mid n \in \mathbb{N}\}$ is a set of recurrence even though $q(n)$ is not LIPR. However, in Proposition 4.1 we give examples of generalized polynomials $q(n)$ for which $\{q(n) \mid n \in \mathbb{N}\}$ are not sets of recurrence.

\section{Some special Results}

We give conditions for when the values of certain generalized polynomials of degree one and two form an averaging set of recurrence. It follows from [9] that these generalized polynomials $q(n)$ have the property P. For most of these generalized polynomials, Theorem 2.3 is not (easily) applicable.

Proposition 4.1. The following conditions hold, where ASR means averaging set of recurrence.

(1) $\left\{\left[[\alpha n] \frac{a}{\alpha}\right] \mid n \in \mathbb{N}\right\}, a \in \mathbb{Q}^{+}, \alpha>0$ irrational, is ASR iff $\alpha<1$ or $a<\frac{\alpha}{[\alpha]}$.

(2) $\left\{\left[[\alpha n] \frac{a}{\alpha}\right]^{2} \mid n \in \mathbb{N}\right\}, a \in \mathbb{Q}^{+}, \alpha>0$ irrational, is ASR iff $\alpha<1$ or $a<\frac{\alpha}{\alpha-1}$.

(3) $\left\{\left[[\alpha n+\theta] \frac{a}{\alpha}\right] \mid n \in \mathbb{N}\right\}, a \in \mathbb{Q}^{+}, \alpha>0$ irrational, is ASR if $0<\theta<\alpha / a$.

(4) $\left\{\left[[\alpha n] \frac{a}{\alpha}+\theta\right] \mid n \in \mathbb{N}\right\}, a \in \mathbb{Q}^{+}, \alpha>0$ irrational, is ASR if $\theta<1$.

(5) $\{[\alpha n+\gamma] \mid n \in \mathbb{N}\}, \alpha$ irrational, is ASR iff there exists $k \in \mathbb{Z}$ such that $\gamma-\alpha k \in[0,1]$.

(6) $\left\{[\alpha n+\gamma]^{2} \mid n \in \mathbb{N}\right\}, \alpha$ irrational, $\gamma \in \mathbb{R}$, is ASR.

(7) $\{[[\alpha n] \beta n] \mid n \in \mathbb{N}\}, \alpha, \beta \in \mathbb{R}$, is $A S R$.

Remarks. (i) The generalized polynomials of (1) and (2) are ASR for any rational value of $\alpha$, while some conditions are needed for those in (3)-(6) if $\alpha$ is rational. 
(ii) Note that if $c>1$ is a rational number such that $\sqrt{c}$ is irrational, then the values of $[[\sqrt{c} n] \sqrt{c}]$ do not form a set of recurrence. However, if $c<\frac{3+\sqrt{5}}{2}$, then $\left\{[[\sqrt{c} n] \sqrt{c}]^{2} \mid n \in \mathbb{N}\right\}$ is an averaging set of recurrence. Hence, $\left\{[[\sqrt{2} n] \sqrt{2}]^{2} \mid n \in\right.$ $\mathbb{N}\}$ is, but $\left\{[[\sqrt{3} n] \sqrt{3}]^{2} \mid n \in \mathbb{N}\right\}$ is not an averaging set of recurrence.

Proof. In the proof of (1)-(4) let $a=l / k$.

(1) If $\alpha<1$ then $\{[\alpha n] \mid n \in \mathbb{N}\}=\mathbb{N}$ so that $\left\{\left[[\alpha n] \frac{a}{\alpha}\right] \mid n \in \mathbb{N}\right\}=\left\{\left[n \frac{a}{\alpha}\right] \mid n \in \mathbb{N}\right\}$ is an averaging set of recurrence by Proposition 2.5.

Let $\alpha>1$. First we show that the condition $\frac{\alpha}{[\alpha]}>\frac{l}{k}$ which is equivalent to $\{\alpha\}>\left(1-\frac{k}{l}\right) \alpha$, is sufficient by using Theorem 3.4. Since the sequence

$$
\left[[\alpha n] \frac{a}{\alpha}\right] \beta=a \beta n-\{\alpha n\} \frac{a}{\alpha} \beta-\left\{a n-\{\alpha n\} \frac{a}{\alpha}\right\} \beta
$$

is uniformly distributed $(\bmod 1)$ if $1, \alpha, \beta$ are rationally independent, we let $\Lambda_{e}=$ $\mathbb{Q}+\alpha \mathbb{Q}$. Let $\Lambda_{0}=\left\{\frac{l_{1}}{k_{1}}, \ldots, \frac{l_{i_{0}}}{k_{i_{0}}}, \beta_{1}, \ldots, \beta_{j_{0}}\right\}$, where $\beta_{i}=\frac{r_{i} \alpha}{s_{i}}$. Let $0<\varepsilon<1-\{\alpha\}$, $k_{0}=\prod_{i} k_{i} s_{i}, r=\prod_{i} r_{i}, n=k k_{0} m+1$ and $S=\left\{n=k k_{0} m+1 \mid\{\alpha m\}<\frac{\varepsilon}{k k_{0}^{2} r l}\right\}$. We have $d(S)>0$. We will show that $S \subset G\left(\Lambda_{0}, \varepsilon\right)$. Now, if $n \in S$, then $\{\alpha n\}=$ $\left\{\alpha m k k_{0}+\alpha\right\}>\{\alpha\}>\left(1-\frac{k}{l}\right) \alpha$ so that $0 \leq \frac{l}{k}\left(1-\{\alpha n\} \frac{1}{\alpha}\right)<\frac{l}{k}\left(1-\left(1-\frac{k}{l}\right) \alpha \frac{1}{\alpha}\right)=1$ and therefore

$$
\left[[\alpha n] \frac{l}{\alpha k}\right]=\left[n \frac{l}{k}-\{\alpha n\} \frac{l}{\alpha k}\right]=l k_{0} m+\left[\frac{l}{k}\left(1-\{\alpha n\} \frac{1}{\alpha}\right)\right]=l k_{0} m .
$$

Hence, $\left[[\alpha n] \frac{a}{\alpha}\right] \frac{l_{i}}{k_{i}}=l k_{0} m \frac{l_{i}}{k_{i}} \equiv 0(\bmod 1)$ and $\left[[\alpha n] \frac{a}{\alpha}\right] \beta_{i}=l k_{0} m \frac{r_{i} \alpha}{s_{i}}$ is $\varepsilon$-close to 0 $(\bmod 1)$ for $n \in S$. Hence, $S \subset G\left(\Lambda_{0}, \varepsilon\right)$ and therefore $d(G)>0$. This completes the proof of the sufficiency.

To show that $\frac{\alpha}{[\alpha]}>\frac{l}{k}$ is necessary, let $l>1$ and $n=m k+r, 0 \leq r \leq k-1$. Then

$$
\left[[\alpha n] \frac{l}{k \alpha}\right]=\left[\frac{l}{k} n+\frac{r l}{k}-\{\alpha n\} \frac{l}{k \alpha}\right]=l m+\left[\frac{l}{k}\left(r-\{\alpha n\} \frac{1}{\alpha}\right)\right]
$$

is divisible by $l$ if and only if $\left[\frac{l}{k}\left(r-\{\alpha n\} \frac{1}{\alpha}\right)\right] \in\{-l, 0\}$, since $\frac{l}{k}\left(r-\{\alpha n\} \frac{1}{\alpha}\right)<$ $\frac{l}{k}(k-1)<l$ for any $n$. Now, if $\left[\frac{l}{k}\left(r-\{\alpha n\} \frac{1}{\alpha}\right)\right]=-l$ then $r=0$ and $\frac{l-1}{l} k \alpha<$ $\{\alpha n\}<1<k \alpha$ which implies that $\alpha k<\frac{l}{l-1}<2$ so that $k=1$ and hence $n=m$ and $1<\alpha<1+\frac{1}{l-1}$. Therefore, $0<1-\frac{\alpha}{l}=\frac{l-1}{l} \alpha-\{\alpha\}<\{\alpha n\}-\{\alpha\}<1-\{\alpha\}<1$ which means that while $\left[[\alpha n] \frac{l}{\alpha k}\right] \frac{1}{l} \equiv 0(\bmod 1),\left\{\left[[\alpha n] \frac{l}{\alpha k}\right] \frac{\alpha k}{l}\right\}=\{\alpha n-\alpha\}$ is bounded away from 0 and 1 . Hence, if $\left\{\left[[\alpha n] \frac{l}{k \alpha}\right] \mid n \in \mathbb{N}\right\}$ is a set recurrence, then $\left[\frac{l}{k}\left(r-\{\alpha n\} \frac{1}{\alpha}\right)\right]=0$ so that $\left[[\alpha n] \frac{l}{\alpha k}\right]=l m$. Therefore $r=1$ and

$$
1>\{\alpha n\}>\left(1-\frac{k}{l}\right) \alpha \text {. }
$$


Now, $\left[[\alpha n] \frac{l}{\alpha k}\right] \frac{\alpha k}{l}=m k \alpha=\alpha n-\alpha$ comes close to $0(\bmod 1)$ if and only if $\{\alpha n\}$ is close to $\{\alpha\}$. So by $(9)$ we need $\{\alpha\}>\left(1-\frac{k}{l}\right) \alpha$. Hence, the condition $\frac{\alpha}{[\alpha]}>\frac{l}{k}$ is necessary.

(2) If $\beta$ is irrational, then $\left[[\alpha n] \frac{a}{\alpha}\right]^{2} \beta$ is uniformly distributed $(\bmod 1)$. Hence, by Theorem 3.4, we need only to check for which $a$ and $\alpha$ the set $\left\{\left[[\alpha n] \frac{a}{\alpha}\right]^{2} \mid n \in\right.$ $\mathbb{N}\}$ is divisible by any $s \in \mathbb{N}$. It follows from the case $n=m k+1$ above that $\left[[\alpha n]_{\frac{l}{k \alpha}}\right]^{2}=l^{2} m^{2}$ when $\{\alpha n\}>\left(1-\frac{k}{l}\right) \alpha$, which is true for a set of $n$ of positive density if and only if $\left(1-\frac{k}{l}\right) \alpha<1$, i.e., if and only if $\frac{l}{k}<\frac{\alpha}{\alpha-1}$.

(3) Let $s \in \mathbb{N}$ and $0<\varepsilon<\frac{\theta}{k s}$. We show that for those $n=k s m$ for which $\{\alpha m\}>1-\varepsilon / l$, both $\left[[\alpha n+\theta] \frac{l}{\alpha k}\right]$ is divisible by $s$ and $\left[[\alpha n+\theta] \frac{l}{\alpha k}\right] \frac{\alpha}{s}$ is $\varepsilon$-close to $0(\bmod 1)$. For under these conditions $\{\alpha n+\theta\}<\theta$ such that $\left[[\alpha n+\theta] \frac{l}{\alpha k}\right]=$ $\left[l s m+\frac{l}{\alpha k}(\theta-\{\alpha n+\theta\})\right]=l s m$ and $\left[[\alpha n+\theta] \frac{l}{\alpha k}\right] \frac{\alpha}{s}=l m \alpha$.

(4) Let $s \in \mathbb{N}$ and $n=k s m$. If $\{\alpha n\}$ and $\{\alpha m\}$ are sufficiently small, then $\left.\left[[\alpha n] \frac{l}{\alpha k}+\theta\right] \frac{1}{s}=m l+\left[\theta-\{\alpha n\} \frac{l}{\alpha k}\right\}\right] \frac{1}{s}=m l \equiv 0(\bmod 1)$ and $\left[[\alpha n] \frac{l}{\alpha k}+\theta\right] \frac{\alpha}{s}=\alpha m l$ is close to $0(\bmod 1)$.

(5) $[\alpha n+\gamma] \beta$ is uniformly distributed $(\bmod 1)$ unless $1, \frac{1}{\alpha}, \beta$ are rationally independent. Observe that $[\alpha n+\gamma] \frac{1}{s} \equiv 0(\bmod 1)$ if and only if $\{(\alpha n+\gamma) / s\}<1 / s$ and is so for a set of $n$ of positive density. Now, writing $n=s m+r, 0 \leq r \leq s-1$, we have

$$
[\alpha n+\gamma] \frac{1}{\alpha s}=n / s+\frac{\gamma}{\alpha s}-\{\alpha n+\gamma\} \frac{1}{\alpha s}=m+r / s+(\gamma-\{\alpha n+\gamma\}) \frac{1}{\alpha s}
$$

which is close to $0(\bmod 1)$ for a set of $n$ of positive density if and only if $\gamma-(k s-$ $r) \alpha \in[0,1]$ for some integer $k$. When $s=1$ this reduces to $\gamma-k \alpha \in[0,1]$ for some $k \in \mathbb{Z}$. Observe that this is also a sufficient condition in this linear case.

(6) Since $[\alpha n+\gamma]^{2} \beta$ is uniformly distributed $(\bmod 1)$ for any irrational $\beta$ and $\{[\alpha n+\gamma] \mid n \in \mathbb{N}\}$ is divisible by any $l \in \mathbb{N},\left\{[\alpha n+\gamma]^{2} \mid n \in \mathbb{N}\right\}$ is an averaging set of recurrence.

(7) If $\alpha^{2} \notin \mathbb{Q}$ and $\beta$ is irrational or $\alpha^{2} \in \mathbb{Q}$ and $\beta$ is rationally independent of $1, \alpha$, then $(\alpha n,[\alpha n] \beta n)$ is uniformly distributed $(\bmod 1)$ [10, Prop. 5.3], so that $\{[[\alpha n] \beta n] \mid n \in \mathbb{N}\}$ is an averaging set of recurrence by Theorem 2.3. This follows also easily if $\beta \in \mathbb{Q}$. Suppose $\alpha=\sqrt{c}, c \in \mathbb{Q}^{+}$, and $\beta=\frac{r}{s} \sqrt{c}+\frac{a}{b}, r, s, a, b \in$ $\mathbb{N}$. We will show that for any $l \in \mathbb{N}$ such that $\left[[\sqrt{c} n]\left(\frac{r}{s} \sqrt{c}+\frac{a}{b}\right) n\right]$ is divisible by $l,\left[[\sqrt{c} n]\left(\frac{r}{s} \sqrt{c}+\frac{a}{b}\right) n\right] \frac{\sqrt{c}}{l}$ comes arbitrary close to $0(\bmod 1)$ for a set of $n$ of positive density. Let $\varepsilon>0$, and let $n=2 l s b m$ and $\frac{2-\varepsilon}{2 l s b}<\{\sqrt{c} m\}<\frac{2}{2 l s b}$. Then $1-\varepsilon<\{\sqrt{c} n\}<1$ and $[\sqrt{c} n]=2 l s b[\sqrt{c} m]+1$, so that by the identity 
$a[b]+b[a]=a b+[a][b]-\{a\}\{b\}$,

$$
\begin{aligned}
{\left[[\sqrt{c} n]\left(\frac{r}{s} \sqrt{c}+\frac{a}{b}\right) n\right] } & =\left[\frac{r c}{2 s} n^{2}+\frac{r}{2 s}[\sqrt{c} n]^{2}-\frac{r}{2 s}\{\sqrt{c} n\}^{2}+[\sqrt{c} n] \frac{a}{b} n\right] \\
& \equiv\left[\frac{r}{2 s}\left(1-\{\sqrt{c} n\}^{2}\right)\right](\bmod l) \\
& =0
\end{aligned}
$$

whenever $1-\{\sqrt{c} n\}^{2}<\frac{2 s}{r}$. Furthermore,

$$
\begin{aligned}
& {\left[[\sqrt{c} n]\left(\frac{r}{s} \sqrt{c}+\frac{a}{b}\right) n\right] \frac{\sqrt{c}}{l}} \\
& \quad=[\sqrt{c} n] \frac{c r}{s l} n+[\sqrt{c} n] \frac{a}{b l} \sqrt{c} n-\left\{[\sqrt{c} n]\left(\frac{r}{s} \sqrt{c}+\frac{a}{b}\right) n\right\} \frac{\sqrt{c}}{l} \\
& \quad \equiv \frac{a}{2 b l}\left(c n^{2}+[\sqrt{c} n]^{2}-\{\sqrt{c} n\}^{2}\right)-\left\{\frac{r}{2 s}\left(c n^{2}+[\sqrt{c} n]^{2}-\{\sqrt{c} n\}^{2}\right)\right\} \frac{\sqrt{c}}{l}(\bmod 1) \\
& \quad \equiv \frac{a}{2 b l}\left(1-\{\sqrt{c} n\}^{2}\right)-\left\{\frac{r}{2 s}\left(1-\{\sqrt{c} n\}^{2}\right)\right\} \frac{\sqrt{c}}{l}(\bmod 1)
\end{aligned}
$$

which, depending on $\varepsilon$, can be chosen arbitrary small.

\section{ACKNOWLEDGMENT}

The authors wish to thank the referee whose penetrating remarks helped us to eliminate some flaws in the original version of the paper.

\section{REFERENCES}

1. V. Bergelson, Sets of Recurrence of $\mathbb{Z}^{m}$-actions and Properties of Sets of Differences in $\mathbb{Z}^{m}$, J. London Math. Soc. (2) 31 (1985), 295-304.

2. V. Bergelson, Ergodic Ramsey Theory, Logic and Combinatorics, ed. S. Simpson, Contemporary Mathematics 65 (1987), 63-87.

3. A. Bertrand-Mathis, Ensembles Intersectifs et Recurrence de Poincare, Israel J. Math. 55 (1986), 184-198.

4. A. H. Forrest, Recurrence in Dynamical Systems: A Combinatorial Approach, Ph.D. Thesis, Ohio State University, 1990.

5. H. Furstenberg, Ergodic behavior of diagonal measures and a theorem of Szemerédi on arithmetic progressions, J. d'Analyse Math. 31 (1977), 204-256.

6. H. Furstenberg, Poincaré Recurrence and Number Theory, Bull. Amer. Math. Soc. 5 (1981), 211-234.

7. H. Furstenberg, Recurrence in Ergodic Theory and Combinatorial Number Theory, Princeton Univ. Press, Princeton, N.J., 1981.

8. H. Furstenberg and B. Weiss, Simultaneous Diophantine Approximation and IP-sets, Acta Arith. 49 (1988), 413-426.

9. I. J. Haaland, Uniform distribution of generalized polynomials, Ph.D. Thesis, Ohio State University, 1992.

10. I. J. Håland, Uniform distribution of generalized polynomials, J. Number Theory 45 (1993), 327-366.

11. I. J. Håland, Uniform distribution of generalized polynomials of the product type, Acta Arith. 67 (1994), 13-27.

12. T. Kamae and M. Mendès France, Van der Corput's difference theorem, Israel J. Math. 31 (1978), 335-342. 
13. A. Sárkösy, On difference sets of sequences of integers I, Acta Math. Acad. Sci. Hungar 31 (1978), 125-149.

Department of Mathematics, The Ohio State University, Columbus, OH 43210

Agder College of Engineering, Grooseveien 36, N-4890 Grimstad, Norway 